\title{
Teoria Fundamentada nos dados para o projeto da mecânica de jogo educacional móvel para adultos mais velhos
}

\author{
Ronan L. R. Ferreira ${ }^{1}$, Lucila Ishitani ${ }^{1}$ \\ ${ }^{1}$ Programa de Pós-graduação em Informática \\ Pontifícia Universidade Católica de Minas Gerais (PUC Minas) \\ Anel Rodoviário Km 23,5 - Rua Walter Ianni, 255 - São Gabriel - 31980-110 \\ Belo Horizonte - MG - Brasil \\ ronan.loschi@gmail.com, lucila@pucminas.br
}

\begin{abstract}
By 2040 about $50 \%$ of the population will be older adults aged 45 years and over. With this increasing life expectancy, there is the need to find alternatives that meet physical and cognitive characteristics of this audience. An alternative is the entertainment union with learning through educational mobile games. This article aims to present a grounded theory containing desirable characteristics for the project of mechanics for these games. Data collection was based on the use of questionnaire, interview, and diary. Data analysis was based on grounded theory. As a result, we present the theory.
\end{abstract}

Resumo. Até 2040 cerca de $50 \%$ da população brasileira será de adultos mais velhos com 45 anos ou mais. Com essa crescente expectativa de vida buscam-se alternativas para as necessidades físicas e cognitivas desse público. Uma alternativa é a união do entretenimento com o aprendizado por meio de jogos móveis educacionais. Esse artigo tem como objetivo principal apresentar uma Teoria Fundamentada nos dados contendo características desejáveis para projetos da mecânica desses jogos. Como metodologia utilizou-se a pesquisa qualitativa. A coleta de dados baseou-se no uso de questionário, entrevista e diário e a análise dos dados, na Teoria Fundamentada. Como resultado alcançado apresenta-se a Teoria.

\section{Introdução}

Em 2010, o Brasil possuía pouco mais de 50 milhões de pessoas com 45 anos ou mais [IBGE 2011], que neste artigo são denominadas de adultos mais velhos. Projeções do Instituto de Pesquisa Econômica Aplicada (IPEA) indicam que, em 2040, o número de pessoas dessa faixa etária será de cerca de 100 milhões, ou $50 \%$ da população brasileira [CAMARANO and KANSO 2009]. Com esse aumento da expectativa de vida, torna-se necessário oferecer mais opções de atividades para os adultos mais velhos, incluindo aprendizagem e lazer. Uma das opções para atender a essa necessidade são os jogos móveis educacionais que possibilitam unir o entretenimento e o aprendizado e podem contribuir para melhorar a qualidade do processo de envelhecimento das pessoas [CHEN et al. 2012].

Um software para ser considerado um jogo precisa ter regras, ter objetivos claros, dar feedbacks ao jogador, permitir o desafio, a competitividade, ser interativo e ter uma narrativa [PRENSKY 2001]. O projeto de um jogo, ou o game design, inclui a tomada 
V Congresso Brasileiro de Informática na Educação (CBIE 2016)

Anais do XXVII Simpósio Brasileiro de Informática na Educação (SBIE 2016)

de centenas ou milhares de decisões [SCHELL 2012]. A mecânica do jogo define o jogo em si e estabelece como será a interação do jogador. Ao estudar as trinta e duas lentes da mecânica de jogos propostas por [SCHELL 2012] e estabelecer a relação dessas lentes com a escala de EGameFlow proposta por [FU et al. 2009] percebe-se uma lacuna nas perguntas que são feitas ao projetista, pois elas não garantem o "olhar" dos projetistas para as necessidades do jogador adulto mais velho.

Esse artigo tem a sua delimitação no projeto da mecânica de jogos móveis educacionais para adultos mais velhos e buscará investigar a seguinte questão norteadora: quais são as características da mecânica de jogos móveis educacionais para adultos mais velhos e como elas se relacionam com a experiência do jogador? Na busca pela resposta, o objetivo principal deste artigo é apresentar uma Teoria Fundamentada nos dados contendo características desejáveis para projetos da mecânica de jogos móveis educacionais para adultos mais velhos.

Nesta pesquisa, utilizou-se a metodologia qualitativa, com características descritivas e de fontes bibliográficas. Os procedimentos de coleta de dados se basearam no uso de instrumentos variados como o questionário, a entrevista e o diário. Os procedimentos de análise dos dados com base na abordagem da Teoria Fundamentada seguiu as diretrizes propostas por [CHARMAZ 2009].

Na Seção 2 são discutidos os trabalhos relacionados com a mecânica de jogos, a aprendizagem do jogador e com o projeto de jogos educacionais para adultos mais velhos. Na Seção 3 é abordada a metodologia do trabalho. Em seguida, na Seção 4 são apresentados os resultados obtidos e, por fim, na Seção 5, as conclusões.

\section{Revisão da literatura}

Nesta seção serão apresentados e discutidos os principais trabalhos identificados na revisão da literatura.

\subsection{Mecânica de jogos}

Das cem lentes propostas por [SCHELL 2012] ${ }^{1}$, a sétima lente "foca" na tétrade elementar, que é composta pelos seguintes elementos: estética, mecânica, história e tecnologia. Essa lente "vê" a possibilidade do projetista considerar cada elemento separadamente e, depois, todos juntos. Este artigo irá considerar separadamente o elemento da mecânica de jogos. Segundo [SCHELL 2012]:

"a mecânica do jogo é a essência do que o jogo realmente é. São as interações e os relacionamentos que permanecem quando a estética, tecnologia e história são removidos."

A mecânica de um jogo é composta por 6 mecânicas principais e suas respectivas lentes, conforme apresentado na Tabela 1:

\footnotetext{
${ }^{1}$ As lentes são pequenos conjuntos de perguntas sobre o projeto, que devem ser respondidas pelo projetista
} 
V Congresso Brasileiro de Informática na Educação (CBIE 2016)

Anais do XXVII Simpósio Brasileiro de Informática na Educação (SBIE 2016)

Tabela 1. Mecânicas do jogo e suas lentes

\begin{tabular}{|l|l|}
\hline \multicolumn{1}{|c|}{ Mecânica: } & \multicolumn{1}{c|}{ Lentes: } \\
\hline 1- Espaço & 21 - Espaço funcional \\
\hline 2- Objetos, atributos e estados & 22 -Espaço dinâmico \\
\hline 3- Ações & 23 - Emersão e 24 - Ação \\
\hline 4- Regras & 25- Objetivos e 26- Regras \\
\hline 5- Habilidade & 27 - Habilidade \\
\hline 6- Probabilidade: & 28- Valor esperado e 29- Oportunidade \\
\hline
\end{tabular}

Fonte: do autor

Essas mecânicas principais devem estar balanceadas e em equilíbrio com outras 23 lentes [SCHELL 2012]. Essas lentes são apresentadas na Tabela 2.

Tabela 2. As 23 lentes para o balanceamento da mecânica de jogos

\begin{tabular}{|l|l|l|l|}
\hline 1. & Lente 30: Equidade & 13. & Lente 42: Simplicidade/complexidade \\
\hline 2. & Lente 31: Desafio & 14. & Lente 43: Elegância \\
\hline 3. & Lente 32: Escolhas significativas & 15. & Lente 44: Caráter \\
\hline 4. & Lente 33: Triangularidade & 16. & Lente 45: Imaginação \\
\hline 5. & Lente 34: Habilidade X sorte & 17. & Lente 46: Economia \\
\hline 6. & Lente 35: Mente e das mãos & 18. & Lente 47: Equilíbrio \\
\hline 7. & Lente 36: Competição & 19. & Lente 48: Acessibilidade \\
\hline 8. & Lente 37: Cooperação & 20. & Lente 49: Progresso visível \\
\hline 9. & Lente 38: Competição X cooperação & 21. & Lente 50: Paralelismo \\
\hline 10. & Lente 39: Tempo & 22. & Lente 51: Pirâmide \\
\hline 11. & Lente 40: Recompensa & 23. & Lente 52: Quebra-cabeça \\
\hline 12. & Lente 41: Punição & & \\
\hline
\end{tabular}

Fonte: do autor

\subsection{Avaliação da aprendizagem do jogador}

[SWEETSER and WYETH 2005] criaram a escala GameFlow, uma escala usada para medir a habilidade do jogador, composta por 8 fatores que são: concentração, desafios, habilidades, controle [autonomia], objetivos claros, comentários, imersão e interação social. A escala GameFlow não descreve adequadamente o aumento do conhecimento, mas serviu de base para o trabalho de [FU et al. 2009], que criaram a escala EGameFlow e incluíram o fator Melhoria do Conhecimento. Segundo os autores a escala EGameFlow pode ser usada como uma referência para o projeto de jogo educacional.

\subsection{Projeto de jogos educacionais para adultos mais velhos}

Em [SANTANA 2015], as autoras fazem um levantamento de características de jogabilidade de jogos educacionais para adultos mais velhos por meio de um estudo de caso com o foco na alfabetização. Os dados foram analisados por meio da Teoria Fundamentada. Dentre os resultados, concluíram que para o sucesso de jogos educacionais no processo de alfabetização é necessário considerar as limitações e as necessidades decorrentes da idade do público-alvo.

No trabalho de [CARNEIRO 2012], o autor apresentou aspectos de usabilidade de mobile learning voltado para usuários com restrições decorrentes da idade. Como resultado, o autor verificou mais de $80 \%$ de interesse no uso de dispositivos móveis por parte dos adultos mais velhos.

Em [FERREIRA and Ishitani 2015], os autores apresentam resultados de uma revisão sistemática da literatura que mostram que são raros os estudos específicos sobre 
V Congresso Brasileiro de Informática na Educação (CBIE 2016)

Anais do XXVII Simpósio Brasileiro de Informática na Educação (SBIE 2016)

projeto de jogos educacionais para adultos mais velhos. Em um desses estudos, os autores apresentam como resultado o Labuta Batuta, um jogo móvel educacional para adultos mais velhos [SILVA et al. 2015]. O jogo é conta com a inclusão de minijogos simples que buscam ensinar o uso dos comandos básicos de navegação em smartphones. A narrativa do jogo traz o cotidiano de um adulto mais velho em sua casa utilizando um smartphone. Primeiramente, o jogador constrói o seu avatar [CHEONG et al. 2011]. Em seguida, o jogador pode navegar pelos cômodos da casa virtual, usando o smartphone para realizar atividades diversas, tais como: tirar foto, enviar mensagens, fazer ligações, instalar e jogar minijogos.

Em [FERREIRA et al. 2015], os autores concluem que os adultos mais velhos podem aprender por meio do uso de um jogo móvel educacional específico para esse público e que eles apreciam essa opção de aprendizagem.

\section{Metodologia}

O método para alcançar os objetivos e responder à questão norteadora desta pesquisa seguiu as seguintes atividades: revisão da literatura; seleção do jogo; seleção dos participantes; coleta de dados e análise de dados pela Teoria Fundamentada.

\subsection{Seleção dos participantes}

Foram selecionados 8 participantes, sendo 6 mulheres e dois homens. Os critérios para participação na pesquisa foram: ter no mínimo 45 anos, consentir na participação voluntária, ser alfabetizado, não possuir deficiência cognitiva, motora ou física que impedisse a realização dos testes ou uso do aparelho celular. Não era necessário possuir um aparelho celular, pois este poderia ser cedido pelo grupo de pesquisa, durante o período da pesquisa. A idade média dos participantes foi igual a 63 anos. O processo de recrutamento seguiu o método bola de neve (Snowball) [BIERNACKI and WALDORF 1981].

O número de participantes está de acordo com a recomendação de se ter um mínimo de 8 em pesquisa utilizando diário [SINGH and MALHOTRA 2013]. Além disso, [CHARMAZ 2009] afirma que, para os propositores da Teoria Fundamentada, amostras pequenas de dados limitados não apresentam problemas.

\subsection{Coleta de dados}

Os instrumentos para coleta de dados foram: um questionário demográfico para conhecer o perfil dos participantes; o Teste de Trilhas [TOMBAUGH 2004] para avaliar a capacidade cognitiva do participante; um diário para o participante registrar com mais detalhes a sua experiência; e uma entrevista semiestruturada.

Para formular as perguntas para o diário e para a entrevista final, percebeu-se a importância de se considerar as 9 lentes da mecânica e as 23 lentes para o seu balanceamento juntamente com os fatores da escala de EGameFlow. Para isso estabeleceu-se por comparação, a correlação entre os fatores da escala EGameFlow [FU et al. 2009] com as 32 lentes que envolvem a mecânica de jogos [SCHELL 2012]. Como resultado dessa correlação foram elaboradas 4 perguntas para o diário e 7 perguntas para a entrevista. $\mathrm{O}$ fator Interação Social foi desconsiderado; pois as perguntas foram direcionadas para um jogo single player. A correlação e as perguntas são apresentadas na Tabela 3. 
V Congresso Brasileiro de Informática na Educação (CBIE 2016)

Anais do XXVII Simpósio Brasileiro de Informática na Educação (SBIE 2016)

Tabela 3. Correlação e definição das perguntas para o diário e a entrevista

\begin{tabular}{|c|c|c|c|}
\hline Fator & $\begin{array}{l}\text { Pergunta da EGameFlow } \\
\text { (para o diário) }\end{array}$ & Lente $\mathbf{n}$ & $\begin{array}{c}\text { Pergunta das lentes } \\
\text { (para a entrevista) }\end{array}$ \\
\hline \multirow{4}{*}{ Concentração } & & 21 & \multirow{2}{*}{$\begin{array}{l}\text { Quais elementos do jogo mantiveram a sua concentração } \\
\text { na aprendizagem e quais atrapalharam ? Explique. }\end{array}$} \\
\hline & & 22 & \\
\hline & & 23 & \multirow{2}{*}{$\begin{array}{l}\text { O que você fazia era interessante para o seu aprendizado } \\
\text { ou gostaria de ter outras ações no jogo? Explique. }\end{array}$} \\
\hline & & 24 & \\
\hline & Total de relações & 4 & \\
\hline \multirow{4}{*}{ Objetivos Claros } & & 25 & \multirow{4}{*}{$\begin{array}{l}\text { Os objetivos do jogo foram apresentados de forma clara? } \\
\text { Explique. }\end{array}$} \\
\hline & & 26 & \\
\hline & & 48 & \\
\hline & & 43 & \\
\hline & Total de relações & 4 & \\
\hline \multirow{3}{*}{ Comentários } & \multirow{3}{*}{$\begin{array}{l}\text { Explique o que achou dos comentários } \\
\text { sobre o seu desempenho durante o jogo. }\end{array}$} & 28 & \\
\hline & & 40 & \\
\hline & & 41 & \\
\hline & Total de relações & 3 & \\
\hline \multirow{7}{*}{ Desafio } & \multirow{7}{*}{$\begin{array}{l}\text { Explique se os desafios do jogo estavam } \\
\text { adequados às suas habilidades. }\end{array}$} & 27 & \multirow{7}{*}{$\begin{array}{l}\text { Como o jogo ajudou você a superar os desafios e como } \\
\text { se sentiu? Explique. }\end{array}$} \\
\hline & & 30 & \\
\hline & & 31 & \\
\hline & & 33 & \\
\hline & & 36 & \\
\hline & & 50 & \\
\hline & & 51 & \\
\hline & Total de relações & 7 & \\
\hline \multirow{4}{*}{ Autonomia } & & 29 & \multirow{4}{*}{$\begin{array}{l}\text { O que um jogo deve ter para você se sentir no controle } \\
\text { e animado a jogar e a aprender? Explique. }\end{array}$} \\
\hline & & 32 & \\
\hline & & 34 & \\
\hline & & 46 & \\
\hline & Total de relações & 4 & \\
\hline \multirow{3}{*}{ Imersão } & \multirow{2}{*}{$\begin{array}{l}\text { Explique se esquecia do tempo ou das } \\
\text { preocupações da vida enquanto jogava. }\end{array}$} & 39 & \\
\hline & & 45 & \\
\hline & Total de relações & 2 & \\
\hline \multirow{7}{*}{$\begin{array}{l}\text { Melhoria do } \\
\text { conhecimento }\end{array}$} & \multirow{6}{*}{ O que você aprendeu? } & 35 & \multirow{6}{*}{$\begin{array}{l}\text { Você ficou motivado a aplicar o que aprendeu no jogo. } \\
\text { Explique. } \\
\text { O que teve no jogo que chamou mais a sua atenção } \\
\text { e foi bom para o seu aprendizado? Explique }\end{array}$} \\
\hline & & 42 & \\
\hline & & 44 & \\
\hline & & 47 & \\
\hline & & 49 & \\
\hline & & 52 & \\
\hline & Total de relações & 6 & \\
\hline
\end{tabular}

Fonte: do autor

O jogo móvel educacional Labuta Batuta foi instalado no celular a ser utilizado pelo participante. Logo após, o pesquisador mostrou ao participante como acessar o jogo e explicou brevemente o seu funcionamento. Em seguida foi explicado aos participantes como preencher o diário. Ressaltou-se que os participantes poderiam deixar de participar a qualquer momento e sem nenhum prejuízo, mas solicitou-se que o jogassem todos os dias. Estabeleceu-se um período de 15 dias para cada participante. Esse tempo foi ideal pois o jogo não apresenta muitos recursos e ficaria repetitivo, se o tempo fosse prolongado. Após esse período os jogadores foram entrevistados sobre a experiência com o jogo. As entrevistas finais foram gravadas. Posteriormente os diários e as entrevistas foram transcritos para análise dos pesquisadores.

A coleta de dados ocorreu em um espaço conhecido pelos participantes e de sua livre escolha. Todos os instrumentos utilizados foram submetidos e aprovados pelo Comitê de Ética em Pesquisa.

\subsection{Análise dos dados coletados}

Os dados foram analisados por meio da Teoria Fundamentada [CHARMAZ 2009]. A Teoria Fundamentada busca atender aos seguintes critérios: ter um ajuste adequado aos 
V Congresso Brasileiro de Informática na Educação (CBIE 2016)

Anais do XXVII Simpósio Brasileiro de Informática na Educação (SBIE 2016)

dados, utilidade, densidade conceitual, durabilidade ao longo do tempo, ser passível de alterações e apresentar poder explicativo. Na prática, o desenvolvimento passa por seis fases de codificação dos dados que são explicados na Tabela 4.

Tabela 4. Fases da Teoria Fundamentada nos dados

\begin{tabular}{|l|l|}
\hline $\begin{array}{l}\text { Fase 1: } \\
\text { Codificação linha a linha: }\end{array}$ & $\begin{array}{l}\text { Permite codificar os dados transcritos } \\
\text { linha a linha [CHARMAZ 2009, p.71-p.87]. }\end{array}$ \\
\hline $\begin{array}{l}\text { Fase 2: } \\
\text { Codificação focal ou focalizada: }\end{array}$ & $\begin{array}{l}\text { Permite-se criar códigos que sintetizam e explicam os } \\
\text { segmentos maiores de dados. [CHARMAZ 2009, p.87-p.90] }\end{array}$ \\
\hline $\begin{array}{l}\text { Fase 3: } \\
\text { Codificação Axial: }\end{array}$ & $\begin{array}{l}\text { Permite criar categorias com os códigos mas frequentes } \\
\text { ou significativos. Códigos menos frequentes ou significativos } \\
\text { são colocados como subcategorias[CHARMAZ 2009, p.92-p.99]. }\end{array}$ \\
\hline $\begin{array}{l}\text { Fase 4: } \\
\text { Codificação teórica: }\end{array}$ & $\begin{array}{l}\text { É um nível sofisticado de codificação e especifica as relações } \\
\text { possíveis entre as categorias desenvolvidas } \\
\text { na codificação focalizada[CHARMAZ 2009, p.92-p.99]. }\end{array}$ \\
\hline $\begin{array}{l}\text { Fase 5: } \\
\text { Redação de memorandos: }\end{array}$ & $\begin{array}{l}\text { Permite analisar os códigos de todas a formas que acorram } \\
\text { [CHARMAZ 2009, p.106-p.133]. Etapa entre a coleta de dados } \\
\text { e a redação dos relatos da pesquisa. }\end{array}$ \\
\hline $\begin{array}{l}\text { Fase 6: } \\
\text { Suficiência teórica: }\end{array}$ & $\begin{array}{l}\text { Ocorre a suficiência teórica quando os dados } \\
\text { não despertam novos códigos, nem revelam novas } \\
\text { propriedades das categorias [CHARMAZ 2009, p.157]. }\end{array}$ \\
\hline
\end{tabular}

Fonte: do autor

\section{Resultados}

Esta seção apresenta os principais resultados relacionados à criação da Teoria Fundamentada nos dados para o projeto da mecânica de jogo educacional móvel para adultos mais velhos.

\subsection{Análise qualitativa de resultados}

Para a análise qualitativa dos dados por meio da Teoria Fundamentada utilizou-se o software "R Qualitative Data Analysis (RQDA)" 2.

Foram gerados no total 316 códigos na codificação linha a linha, sendo 78 códigos com os diários e com as observações e 238 com as entrevistas.

Analisando os 316 códigos gerados na codificação linha a linha foram gerados 81 códigos na codificação focalizada, considerando os que permitiram sintetizar e explicar os segmentos maiores de dados.

Na codificação Axial foram geradas 15 categorias considerando os códigos mais significativos e/ou frequentes da codificação focalizada. E os 81 códigos foram relacionadas às categorias criadas, como subcategorias. Observou-se que das 15 categorias, 6 já estavam contempladas na literatura, sendo elas: Desafios, Feedbacks, Melhoria do conhecimento, Objetivos Claros, Imersão, Autonomia [FU et al. 2009] e [SCHELL 2012]. As outras 9 categorias, que foram consideradas novas, são: Minijogos, Avaliação do desempenho, Evoluindo no jogo, Entretenimento e aprendizagem, Niveis, Uso de dispositivos móveis, Concentração na aprendizagem, Gostando de jogar e Links externos. A descoberta dessas 9 categorias reforçam a lacuna na literatura pesquisada e surgem como novas características para o projeto da mecânica de jogos móveis educacionais para adultos mais velhos.

\footnotetext{
${ }^{2}$ https://www.R-project.org/. Acesso em: fev de 2016.
} 
V Congresso Brasileiro de Informática na Educação (CBIE 2016)

Anais do XXVII Simpósio Brasileiro de Informática na Educação (SBIE 2016)

A codificação teórica permitiu especificar as relações possíveis entre as categorias desenvolvidas. As relações foram geradas a partir dos relatos dos participantes. Por exemplo, nos relatos a seguir, pode-se observar algumas dessas relações:

Relação I: Melhoria do conhecimento afeta o Entretenimento e aprendizagem por meio da subcategoria "aprendendo por meio do jogo".

“(...) se fala que eu vou precisar mexer no Face eu já sei, está mais direto; e antes eu tinha dificuldades, chamava alguém para me ajudar, agora não

$<$ Melhoria do Conhecimento $>$, depois que eu aprendi no jogo que eu comecei a associar e aprender ué, aprendi melhor $<$ Entretenimento $\boldsymbol{e}$ aprendizagem $>$." [Arcia, P6, entrevista]

Relação II: Minijogos afeta positivamente a Melhoria do Conhecimento por meio da subcategoria "Achando os minijogos interessantes para concentrar-se na aprendizagem".

“(...). Mas sem dúvida é esse ai do pergunta e responde (Eu Te Pergunto) $<$ Minijogos $>$, esse ai me fez evoluir, destacou para mim, para o aprendizado; nesse que eu aprendi mais, nesse ai $<$ Melhoria do Conhecimento $>$ ".[Lina, P7, entrevista]

Relação III: Objetivos claros afeta Gostando de jogar por meio da subcategoria "entendendo claramente os objetivos do jogo".

“(...) foi tudo muito bem explicadinho, teve assim nada de dificuldade, eu não achei não <objetivos claros $>$, eu achei que foi legal $<$ Gostando de jogar >. (...).” [Equel, P3, entrevista]

O conjunto de códigos teóricos gerados e suas relações são apresentados na Tabela 5 .

Tabela 5. Codigos teóricos e suas relações

\begin{tabular}{|l|l|}
\hline $\begin{array}{c}\text { Código } \\
\text { teórioco }\end{array}$ & \multicolumn{1}{c|}{ Relações } \\
\hline 1 & Objetivos claros afeta: Gostando de jogar. \\
\hline 2 & Evoluindo no jogo afeta: Gostando de jogar, Melhoria do conhecimento e Desafios. \\
\hline 3 & Concentração na aprendizagem afeta: Gostando de Jogar. \\
\hline 4 & Imersão afeta: Evoluindo no jogo e Entretenimento e aprendizagem. \\
\hline 5 & Desafios afetam: melhoria do conhecimento, Níveis e Gostando de jogar. \\
\hline 6 & $\begin{array}{l}\text { Entretenimento e aprendizagem afeta: Melhoria do conhecimento, os Níveis, } \\
\text { Uso de dispositivos móveis, Gostando de jogar e Links externos. }\end{array}$ \\
\hline 7 & $\begin{array}{l}\text { Minijogos afeta: Melhoria do conhecimento, Uso de dispositivos móveis, Gostando de jogar } \\
\text { e Entretenimento e aprendizagem. }\end{array}$ \\
\hline 8 & $\begin{array}{l}\text { Gostando de jogar afeta: Melhoria do conhecimento, Entretenimento e aprendizagem, } \\
\text { Evoluindo no jogo, Níveis, Uso de dispositivos móveis e a Imersão. }\end{array}$ \\
\hline 9 & Níveis afetam: Gostando de jogar, Entretenimento e aprendizagem e os Desafios. \\
\hline 10 & Feedbacks afetam: Gostando de jogar e a Avaliação do desempenho. \\
\hline 11 & Uso de dispositivos móveis afeta: Entretenimento e aprendizagem. \\
\hline 12 & Autonomia afeta: Concentração na aprendizagem, a Melhoria do Conhecimento e o Gostando de jogar. \\
\hline 13 & Avaliação do desempenho afeta: Evoluindo no jogo. \\
\hline 14 & $\begin{array}{l}\text { Melhoria do conhecimento afeta: Entretenimento e aprendizagem, Uso de dispositivos móveis, } \\
\text { Objetivos claros, Desafios,Níveis e Gostando de jogar. }\end{array}$ \\
\hline
\end{tabular}

Fonte: do autor

As relações entre os códigos teóricos podem ser observadas na Figura 1. Gostando de jogar, Melhoria do conhecimento e Entretenimento e aprendizagem foram as 
V Congresso Brasileiro de Informática na Educação (CBIE 2016)

Anais do XXVII Simpósio Brasileiro de Informática na Educação (SBIE 2016)

categorias que mais afetaram e mais foram afetadas por outras categorias. Gostando de jogar afetou e foi afetada por 10 categorias; Entretenimento e aprendizagem afetou $5 \mathrm{e}$ foi afetada por 7 e Melhoria do conhecimento afetou 5 e foi afetada por 6 .

Figura 1. Modelo teórico das relações entre as categorias

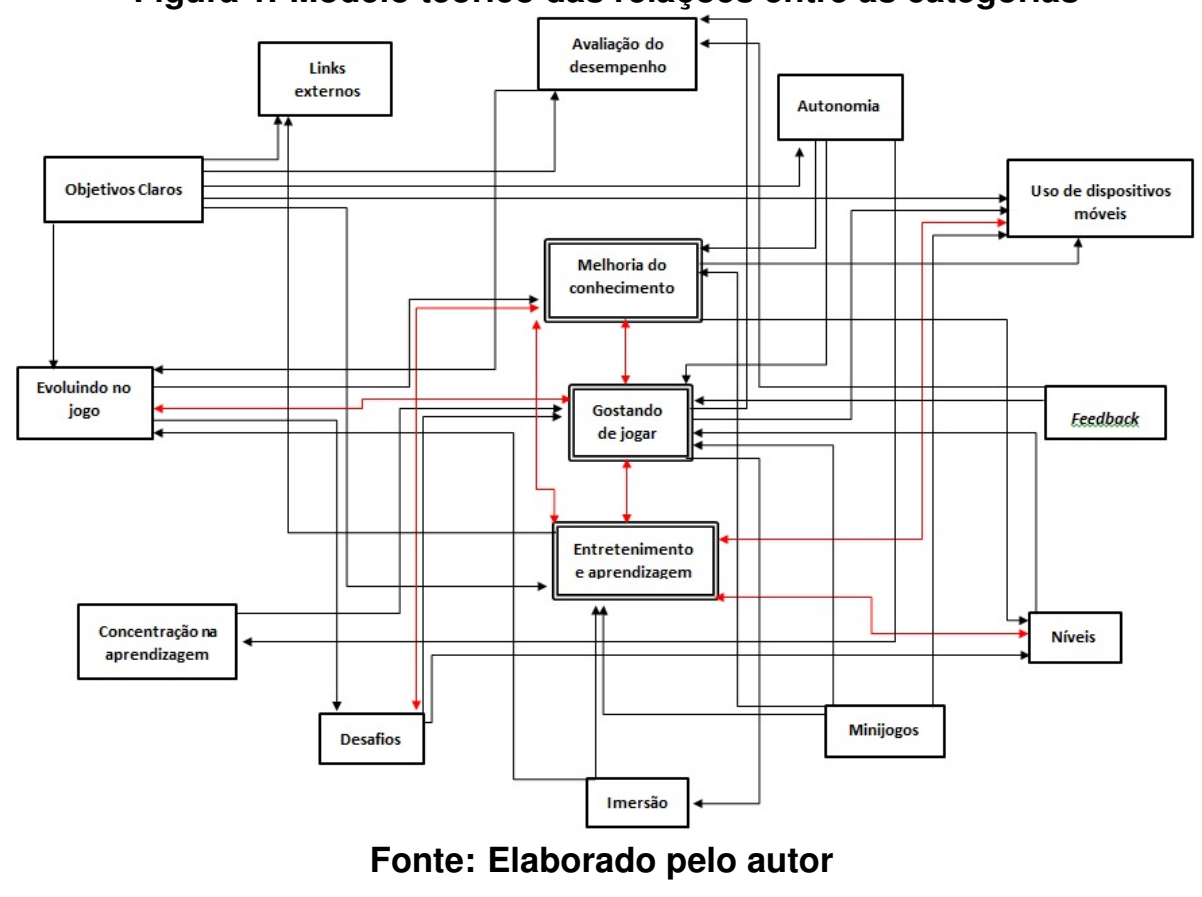

\subsection{A construção da Teoria Fundamentada nos Dados}

As relações estabelecidas e o modelo teórico serviram de base para a construção da Teoria Fundamentada nos dados com características para o projeto da mecânica de jogo educacional móvel para adultos mais velhos, que é apresentada no texto que se segue.

As categorias Gostando de jogar, Entretenimento e aprendizagem e Melhoria do conhecimento são as categorias centrais do modelo teórico pois estabeleceram o maior número de relações e foram as que mais afetaram e foram afetadas na opinião dos jogadores (adultos mais velhos). Isso demonstra que quando esse público gosta de jogar um jogo móvel educacional ele valoriza a união do entretenimento com o aprendizado e entende que essa união leva à melhoria do conhecimento que favorece a imersão e a aprendizagem continuada. Portanto o projeto da mecânica de jogos móveis educacionais para adultos mais velhos deve considerar essas relações como características principais.

As relações da categoria Uso de dispositivos móveis mostram que, ao utilizar um dispositivo móvel para jogar e aprender, o adulto mais velho desperta o seu interesse pelo uso das tecnologias móveis permitindo o seu entretenimento e a aprendizagem. Assim o uso de dispositivos móveis é também uma característica a ser considerada. A categoria Objetivos claro e suas relações mostram que ao entender claramente os objetivos do jogo móvel educacional o adulto mais velho passa a compreender melhor o jogo e como jogar. Com isso eles alcançam os objetivos do jogo e valorizam a oportunidade de se divertir e de aprender ao mesmo tempo, além de vencerem as barreiras de uso das tecnologias móveis. Logo objetivos claros é também uma característica para a mecânica de jogos móveis educacionais. 
V Congresso Brasileiro de Informática na Educação (CBIE 2016)

Anais do XXVII Simpósio Brasileiro de Informática na Educação (SBIE 2016)

A categoria Evoluindo no jogo, com suas relações, mostra que à medida em que o adulto mais velho evoluir no jogo ele passará a ter mais interesse, curiosidades e a querer vencer os desafios do jogo. E esses desafios precisam estar adequados às habilidades do jogador [SWEETSER and WYETH 2005] e preferencialmente distribuídos em níveis. Assim, permitir que o adulto mais velho possa evoluir aos poucos no jogo, equilibrando os desafios com as habilidades do jogador é mais uma característica que tornará o jogo mais interessante para esse público. Caso contrário, a falta desse equilíbrio pode frustrar o jogador e fazer com que ele pare de jogar.

Para os adultos mais velhos os minijogos ajudam na concentração, motivam a aprendizagem do jogador e possibilitam o melhor uso do dispositivo móvel utilizado para jogar. Portanto, as relações da categoria Minijogo mostram outra característica para o projeto da mecânica de jogos móveis educacionais para adultos mais velhos que é incluir minijogos que despertem no jogador a atenção e a curiosidade por descobrir o que cada minijogo tem a oferecer e que também lhes permitam avaliar o seu desempenho no jogo.

As relações da categoria Comentários (Feedbacks) mostram que esses comentários incentivam os jogadores e influenciam para gostarem do jogo e para continuarem jogando, por isso esses comentários devem ser bem elaborados e considerados mais uma característica.

Outra característica para que o jogador sinta-se no controle do jogo é considerar suas limitações físicas e/ou cognitivas. Uma alternativa é inserir links externos para estimular o adulto mais velho a exercitar outros movimentos e conhecer conteúdos extras.

\section{Conclusões}

Essa pesquisa teve como objetivo a construção de uma Teoria Fundamentada nos dados contendo as características que servirão como base para projetos da mecânica de jogos móveis educacionais para adultos mais velhos. Para isso utilizou-se a pesquisa qualitativa e instrumentos variados como o questionário, a entrevista e o diário. Para a análise dos dados utilizou-se a Teoria Fundamentada.

Dentre as contribuições destaca-se a descoberta de 9 novas categorias que são: Minijogos, Avaliação do desempenho, Evoluindo no jogo, Entretenimento $e$ aprendizagem, Níveis, Uso de dispositivos móveis, Concentração na aprendizagem, Gostando de jogar e Links externos. Essas descobertas somam aos trabalhos de [SWEETSER and WYETH 2005] e de [SCHELL 2012] para projetos da mecânica de jogo móvel educacional para adultos mais velhos.

Do ponto de vista dos participantes o trecho abaixo reforça a importância do jogo educacional móvel para o adulto mais velho:

"Eu acho é, para mim foi um prazer imenso participar (...), porque eu nunca usei internet, falar a verdade, nunca usei sabe, então foi um aprendizado para mim ótimo e eu agradeço isso, porque me despertou a vontade de usar (...) ainda vou querer um celular desses de com mais né, com, mais evoluído para usar né”.

Sugere-se que trabalhos futuros busquem: desenvolver um jogo móvel educacional ampliando seus recursos e seguindo as características da mecânica do jogo presentes na Teoria Fundamentada proposta nesse artigo. E em seguida testar o novo jogo com 
V Congresso Brasileiro de Informática na Educação (CBIE 2016)

Anais do XXVII Simpósio Brasileiro de Informática na Educação (SBIE 2016)

adultos mais velhos para validar a Teoria comparando os resultados com outros jogos educacionais que não seguiram as mesmas características propostas na Teoria.

\section{Agradecimentos}

Esta pesquisa recebeu apoio financeiro do CNPq (475311/2012-4) e da FAPEMIG.

\section{Referências}

BIERNACKI, P. and WALDORF, D. (1981). Snowball sampling: Problems and techniques of chain referral sampling. Sociological Methods Research, 10(2):141-163.

CAMARANO, A. A. and KANSO, S. (2009). Perspectivas de crescimento para a população brasileira: velhos e novos resultados. IPEA, Rio de Janeiro.

CARNEIRO, R. V. (2012). Aspectos de usabilidade de mobile learning voltado para usuários com restrições decorrentes da idade. In Dissertação.

CHARMAZ, K. (2009). A Construção da Teoria Fundamentada: Guia prático para análise qualitativa. Artmed, Porto Alegre.

CHEN, S.-T. et al. (2012). Effects of improvement on s. a.: Developing appropriate somatosensory video game. Turkish Online Journal of Educational Technology.

CHEONG, W. L., Jung, Y., and Theng, Y.-L. (2011). Avatar: A virtual face for the elderly. In Proceedings of 10th International Conference on VRC, VRCAI'11, pages 491-498.

FERREIRA, R. L. et al. (2015). Aprendizagem do uso de smartphones por adultos mais velhos mediada por jogo educacional. Anais do XXVI SBGames.

FERREIRA, R. L. and Ishitani, L. (2015). Jogos educacionais digitais para idosos: uma revisão sistemática de literatura. Anais do XXVI SBIE.

FU, F.-L., Su, R.-C., and Yu, S.-C. (2009). Egameflow: A scale to measure learners' enjoyment of e-learning games. Comput. Educ., 52(1):101-112.

IBGE (2011). Censo Demográfico 2010 - Características da população e dos domicílios. Instituto Brasileiro de Geografia e Estatística - IBGE, Rio de Janeiro.

PRENSKY (2001). Kthe adult learner Website. Acessado http: / / goo.gl/r8GU0 9 em 2016. Accessed jan 2016.

SANTANA, R. C. (2015). Levantamento de características de jogabilidade de jogos educacionais para adultos mais velhos: um estudo de caso com foco na alfabetização. In Dissertação.

SCHELL, J. (2012). A arte de game design: o livro original. Rio de Janeiro: Elsevier.

SILVA, R. et al. (2015). Labuta batuta: um jogo educacional móvel para adultos mais velhos. Anais do XXVI SBGames.

SINGH, A. and MALHOTRA, S. (2013). A researcher's guide to running diary studies. In Proceedings of 11th Asia Pacific Conference on IHC, APCHI '13, pages 296-300.

SWEETSER, P. and WYETH, P. (2005). Gameflow: A model for evaluating player enjoyment in games. Comput. Entertain., 3(3):3-3.

TOMBAUGH, T. N. (2004). Trail making test a and b: Normative data stratified by age and education. Archives of Clinical Neuropsychology, 19(2):203 - 214. 